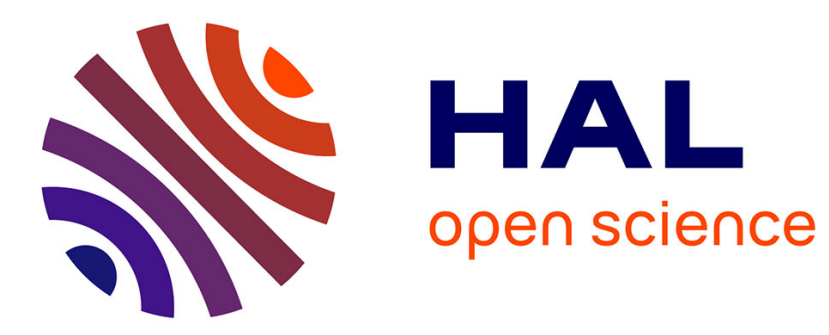

\title{
Design and Modeling of a Polymer Force Sensor
}

Nitish Kumar, Olivier Piccin, Laurence Meylheuc, Laurent Barbe, Bernard Bayle

\section{To cite this version:}

Nitish Kumar, Olivier Piccin, Laurence Meylheuc, Laurent Barbe, Bernard Bayle. Design and Modeling of a Polymer Force Sensor. IEEE/ASME Transactions on Mechatronics, 2015, 21 (1), pp.555-564. 10.1109/TMECH.2015.2448662 . hal-02320641

\section{HAL Id: hal-02320641 \\ https://hal.science/hal-02320641}

Submitted on 18 Oct 2019

HAL is a multi-disciplinary open access archive for the deposit and dissemination of scientific research documents, whether they are published or not. The documents may come from teaching and research institutions in France or abroad, or from public or private research centers.
L'archive ouverte pluridisciplinaire HAL, est destinée au dépôt et à la diffusion de documents scientifiques de niveau recherche, publiés ou non, émanant des établissements d'enseignement et de recherche français ou étrangers, des laboratoires publics ou privés. 


\title{
Design and Modeling of a Polymer Force Sensor
}

\author{
Nitish Kumar, Olivier Piccin, Laurence Meylheuc, Laurent Barbé, Bernard Bayle
}

\begin{abstract}
This article presents the design, modeling, force correction strategies and experimental validation of a force sensor designed for robotized medical applications. The proposed sensor offers a new solution for force measurement in the presence of specific constraints such as medical imaging transparency, limited size, satisfactory rigidity and measurement performance. More specifically, the presented prototype has been purposely adapted to comply with the requirements of needle insertion applications, in the context of interventional radiology. A systematic viscoelastic model identification method is discussed for choosing the best time-dependent model for the force sensor. A novel compensation law is proposed based on the chosen model to correct for the viscoelastic effects of the utilized polymer material. The developed compensation law is inexpensive, stable to noise and can be applied in real-time to the sensor signal. A comparative assessment of the experimental results, obtained from quasi-static to dynamic experiments including harmonic analysis, shows the efficacy of the proposed compensation law, as compared to calibration with static gain and without compensation. The improvement in the sensor response results in decreased hysteresis levels and increased bandwidth, which are improved by more than a factor of 4 .
\end{abstract}

Index Terms-Sensors and Sensing Systems, Medical Robotics, Mechatronics, Modeling, Compensation

\section{INTRODUCTION}

$\mathbf{R}$ OBOTICS has increasingly found applications in several medical specialties [1] ranging from assisting the surgeons in various procedures to patient rehabilitation or medical implants. There has been lot of work in the area of computer-integrated surgery and robotic assistants [2], [3] which help practitioners within the surgical workflow by improving accuracy, increasing repeatability or reducing fatigue. While minimally invasive surgery (MIS) offers advantages in terms of reduced pain and patient recovery time, surgeons face the disadvantage of decreased access to the patient. In interventional radiology or emerging hybrid surgery, MRI, Xray $\mathrm{CT}$ and fluoroscopy, ultrasound are some of the imaging modalities utilized for having improved visualization of the inaccessible organs and tissues. During common MIS procedures like biopsies, the prime objective of the surgeon is to insert a needle to reach a target position. Apart from visual feedback, the fine perception of interaction forces might help the practitioner in his/her gestures and decisions, as often discussed in literature [4], [5], [6]. This feedback to the surgeon can help him/her to better detect the transitions between different organs or tissue layers of different density. This especially is the case when no real-time imaging is available. The key events, such as tissue rupture corresponding

N. Kumar, O. Piccin, L. Meylheuc, L. Barbé and B. Bayle are with the automatic, vision and robotics team of the ICube laboratory, University of Strasbourg and Image-guided Hybrid Surgery Institute (IHU Strasbourg) France. Email: n. kumareunistra.fr to sudden decrease in the level of interaction forces, can be immediately detected and possibly rendered to the practitioner. Therefore force sensors are recommended for providing such haptic force feedback to augment the visual display.

The focus of this paper is the medical specialty of interventional radiology where radiologists use CT scanner images for various interventions like tumor ablations or biopsies. The repeated exposure to X-rays, though useful for medical diagnosis and treatment, is harmful for the medical staff due to the ionizing nature of the X-rays. The resulting need for a robotic assistant in the context of interventional radiology comes from protecting the radiologist and the staff. Teleoperation scenarios [7] have been proposed and implemented for distancing the radiologist from the high exposure area. A master robot is operated by the radiologist, which teleoperates a slave robot directly interacting with the patient as described in the schematic Fig. 1. A similar scenario could be envisaged for MRI guided procedures, where teleoperation is not justified by staff protection but by the very difficult access to the patient.

As the radiologist is not in direct contact with the patient, haptic feedback is needed to help him in a bilateral teleoperation scenario [8]. The force sensor which forms a part of the slave robotic assistant is used to measure actual interaction forces, so as to provide haptic feedback to the radiologist. The X-ray imaging produces artifacts in the image due to presence of predominantly metallic elements. This degrades the image quality and may render it useless for carrying out the medical procedure. Most of the existing works on robotic assistants for CT guided needle insertion interventions [9], [10], [11] do not include a force sensor or other means for providing haptic force feedback. The only custom designed solution for such a use has been developed in the work of [12], which includes a metallic force sensor. However to remain compatible with CT imaging and to avoid metallic elements in the imaging plane, this force sensor is placed away from the needle axis and involves intermediate drive train mechanism for force transmission. To have a direct measurement of the needle insertion forces and minimize friction effects, it is preferable to have the force sensor close to the patient body and aligned with the insertion axis. The direct utilization of off-the-shelf commercial force sensors is not possible due to limitations imposed by the imaging modalities like CT or MRI on usable materials. Since MRI uses high magnetic fields, no ferromagnetic material can be present within its close surroundings. The constraints of MRI are more strict than those of CT, which does not require the total elimination of electrical components. For ensuring compatibility with CT imaging, polymers can be used as materials for the body of the force sensor. Few works [13], [14], [15], [16] can be found on MRI compatible force sensors utilizing polymers as a material 


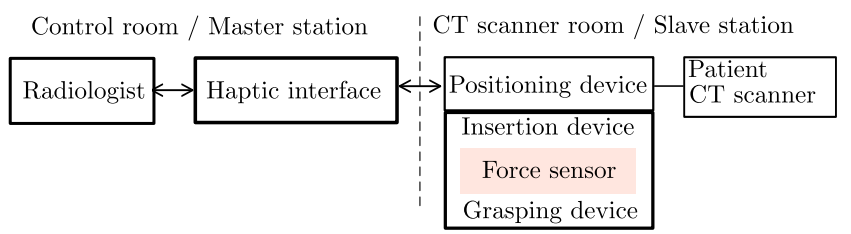

Fig. 1: The force sensor within the general layout of the teleoperated percutaneous procedures. Reprinted from [22].

They all have a sensing principle based on optics to avoid any electrical components. A uniaxial force sensor using optical fibers compatible with ultrasound imaging [17] was developed for minimally invasive beating heart surgery. The range of the measured forces and the degrees of freedom (DOF) of these sensors vary depending on the medical intervention. That is why it becomes necessary to adapt the force sensor design according to the utilized imaging technology and targeted medical intervention. Furthermore, utilizing a polymer as a force sensor material introduces viscoelastic effects, which can significantly affect its performance if they are not properly modeled and accounted for. The above papers do report hysteresis but no modeling and compensation is proposed. The development and modeling of a triaxial force sensor for MRI compatibility is proposed in [18]. To compensate for the hysteresis effects in piezoelectric actuators, an inverse ratedependent model using play operator of Prandtl-Ishlinskii [19] has been used. This approach uses a mathematical model to describe the training sets of static hysteresis data. It does successfully compensate and correct for errors in the hysteresis curve. However, hysteresis is one of the resulting effects of the inherent viscoelasticity of the polymer material. This approach does not discuss the efficacy of the compensation model with respect to other time-dependent effects of the viscoelasticity leading to drift in the sensor signal value with respect to time. Also this compensation model does not discuss the effects on the dynamic response of the force sensor and the results are discussed for static data only. The time component is missing from the data sets. This kind of model could potentially be less stable to noise, more complicated to model and sensitive to the direction of loading due to the non-linear nature of the model. A physical linear viscoelastic model involving springs and dashpots such as found in work of [20], [21] for modeling of actuators can provide a good approximation of the inherent viscoelastic effects including hysteresis and time-dependent effects. This viscoelastic model can then be used to formulate a compensation law which can be applied to both static and dynamic response of the force sensor.

In the present work, the contributions are twofold. First this paper presents the design and modeling of a force sensor based on polymer material and adapted for needle insertion procedures guided by $\mathrm{CT}$ imaging. It has been developed, in the context of interventional radiology, in order to provide haptic force feedback to the radiologists. This article extends the previous work by the authors [22] , where the design and preliminary assessment of this force sensor were taken up, assuming only an elastic model. Secondly a procedure for static and dynamic compensation of the viscoelastic effects including hysteresis, time-dependent drifts has been proposed, which is applicable to sensors having a polymer body. A compensation law derived from the viscoelastic model of the force sensor has been developed, which is stable to noise, computationally inexpensive and applicable to the sensor signal both offline and in real-time for experimental assessment. This paper is divided into five sections including conclusions. Section II lists the specific requirements arising from the targeted application and presents the force sensor. Section III describes the experimental setups and results of the quasistatic experiments. In section IV, a linear viscoelastic model identification method and the developed compensation law are described. Finally, section V presents the characterization of the force sensor with compensation, when subjected to dynamic loadings.

\section{Force SENSOR DESCRIPTION}

\section{A. Requirements for the Force Sensor}

1) Size: The designed force sensor is an integral part of a needle insertion robotic assistant which may take the form of a table-mounted [9] or patient-mounted [23] system. Mounting the robotic assistant on the patient leads to a more compact design with smaller footprint. After the introduction of a patient in the tunnel of a CT scanner, the available height is less than $300 \mathrm{~mm}$ [10]. This constrains the size of the robotic assistant and all of its components. As a result, the force sensor has to be as compact as possible and a maximum volume of $25 \times 25 \times 25 \mathrm{~mm}$ has been put as the constraint on the size of the force sensor.

2) Force measurement performance: There could be scenarios where the force sensor does not lie directly along the axis of the insertion and involves intermediate mechanisms for transmission of the insertion forces. In such cases, the effect of friction in the transmission mechanism and the nature of the underlying mechanism itself changes the force signal. This strategy does not ensure a good transmission of the actual insertion force. It is preferable to have the force sensor lying along the insertion axis and to avoid the use of intermediate mechanisms. This would in turn require that the force sensor body has a through hole at its center along the insertion axis for allowing the needle to pass through.

3) Material: CT scanner compatibility does not require a total elimination of the metallic parts. However, adequate image acquisition requires the imaging plane of the CT scanner to be free from dense metallic parts. As the force sensor geometry needs to be defined so as to surround the needle barrel, its constitutive materials should be radiolucent. Hence, a polymer based material is chosen for fabrication of the prototype.

4) DOF of the sensor: The work of Maurin et al. [24], also co-authors of this article, studied the in vivo evolution of forces during needle insertion in an anesthetized swine. The measure of forces and moments along the transverse directions were found to be in the order of $10^{-3} \mathrm{~N}$ and $10^{-3} \mathrm{Nmm}$, respectively, which is very small compared to the order of magnitude of the axial forces/moments. Hence, the component of the force in 
the axial direction is sufficient to give the radiologist the main feedback for detecting the keys events such as tissue rupture or changes in the tissue density. Hence, a 1-DOF sensor is sought to be designed, which can measure the axial forces while rejecting the moment disturbances along the same axis.

5) Specifications: The study in [24] also showed that the magnitude of the axial forces during needle insertion can go up to $4 \mathrm{~N}$ with skin puncture. Hence, the nominal rated force for the force sensor has been kept as $10 \mathrm{~N}$. For the purpose of haptic feedback, high levels of accuracy is not required though resolution and bandwidth of the force sensor should be enough to capture the haptic information. For direct access to the liver, the axial force measurement can go up to $0.7 \mathrm{~N}$ with a change of $0.1 \mathrm{~N}$ during the event of capsule puncture. The work of Grana et al. [25] revealed after a frequency analysis that the power of insertion forces signal is concentrated in the frequency range of $[0 ; 3] \mathrm{Hz}$. Hence, a bandwidth up to $3 \mathrm{~Hz}$ would ensure that relevant frequency content in the insertion force signal is not attenuated. The displacement of the force sensor in the axial direction has to be very small (lower than $0.5 \mathrm{~mm}$ ) so as not to limit the accuracy of the needle insertion procedure. A high rotational stiffness is required for rejecting the axial moment load disturbances. Finally, hysteresis due to viscoelastic effects in the polymer based material has to be minimized.

6) Sensing principle: The sensing principles based on light and optical fibers have already been discussed in the introduction. The optical fibers are difficult to pass through the moving parts of the robotic assistant and require an extra amplification mechanism as very small deformations cannot be measured directly. A three axis, CT compatible force sensor based on strain gages [12] has been developed for integration with a robotic assistant but this embodiment utilizes intermediate mechanisms for transmission of the insertion forces. Moreover, electric wires are easier to route on the moving parts than optical fibers and do not alter the CT image quality. Very small strains can be measured without the need for any amplification mechanism. Finally, strain gages have the advantages of smaller size and easy integration to a flexible element. Hence, force sensing principle based on strain gages is selected in this work.

\section{B. Force Sensor Design}

Force sensors using strain gages always involve one or several parts, the deformations of which serve to reconstruct the applied input forces. The selection for one or more elastic elements can be performed using the techniques developed in the field of compliant mechanism design. The two main methods for compliant mechanism design are the rigid-body replacement synthesis and the synthesis accounting for energy storage of the mechanisms [26]. In the first approach, the designer first takes advantage from the numerous methods available from classical rigid-body mechanism synthesis before transforming some parts of the mechanism candidates into flexible members. The proposed force sensor architecture has been elaborated following this approach. The passage of the needle through the body of the force sensor as explained

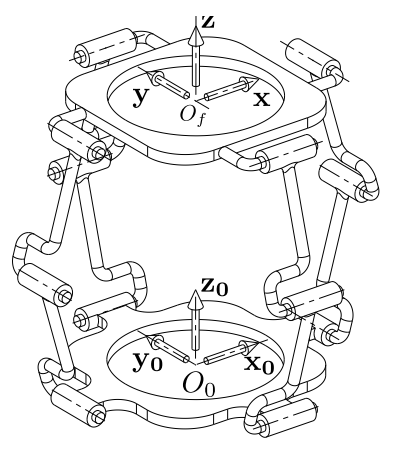

(a)

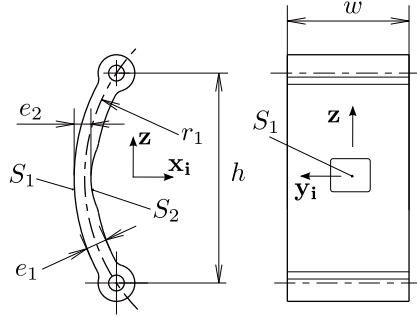

(b)
Fig. 2: Conversion from rigid body to compliant model (a) 4-leg Sarrus mechanism (b) Geometry of a flexure member.

TABLE I: Simulation results under loading conditions: axial force $-25 \mathbf{z ~ N}$, axial moment $-25 \mathbf{z} \mathrm{mNmm}$.

\begin{tabular}{l|l}
\hline Axial displacement along $\mathbf{z}$ & $0.16 \mathrm{~mm}$ \\
Axial stiffness & $156 \mathrm{~N} / \mathrm{mm}$ \\
\hline Platform rotation about $\left(O_{f}, \mathbf{z}\right)$ & $3.710^{-4} \mathrm{rad}$ \\
Torsional stiffness & $6.710^{5} \mathrm{mN} \cdot \mathrm{m} / \mathrm{rad}$ \\
\hline
\end{tabular}

in paragraph II-A2 is a key requirement for ensuring the best force transmission. The Sarrus mechanism fulfills the aforementioned constraints and its simplest form consists in a 6R closed loop linkage that can be seen as an overconstrained parallel mechanism with two $3 \mathrm{R}$ legs. The preferred variant for the application has been selected with four legs [22], as depicted in Fig. 2(a), to enhance the overall mechanism rigidity.

Following the rigid-body replacement synthesis approach, a compliant Sarrus mechanism (CSM) has been elaborated by replacing the middle $\mathrm{R}$ joint of each leg by a flexible curved plate geometry, as depicted in Fig. 2(b). An exploded CAD view of the CSM is given in Fig. 3(a). When submitted to the axial loading along $-\mathbf{z}$, the maximum tensile and compressive strains develop at the central part of the curved plates denoted $S_{1}$ and $S_{2}$ in Fig. 2(b). Therefore, $S_{1}$ and $S_{2}$ are the surfaces where the strain gages are to be bonded. A FEM (finite element method) simulation setup has been used for studying the behavior of the CSM when submitted to different loading cases with various geometric configurations of the flexure elements. The simulation results have put forward a design candidate with the design set of parameters $\left(e_{1}=2.0 \mathrm{~mm}\right.$, $r_{1}=18 \mathrm{~mm}$ ) (see [22] for more details). The results of the calculated displacement and stiffness of the CSM are summarized in Table I. Under the axial moment load, the maximum strains developed at $S_{1}$ and $S_{2}$ surfaces was of the order of $10^{-7}$ compared to $10^{-3}$ developed under axial force loading.

The force sensor prototype pictured in Fig. 3(b) was fabricated with a Connex 350 rapid prototyping machine that can produce parts in different digital materials featuring mechanical stiffness properties ranging from rather rigid to rubberlike materials. Any of these shades of polymer material has a viscoelastic behavior and two types of material were selected 


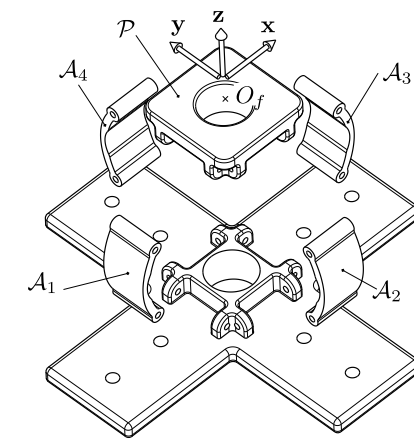

(a)

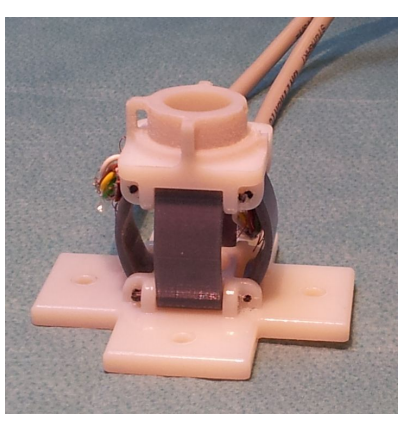

(b)
Fig. 3: Compliant Sarrus mechanism CAD and prototype (a) Exploded view (b) Fabricated prototype.

for the prototype depending on the function of each part: the base and the platform which are assumed to be rigid were constructed using the stiffer digital material offered by the machine whereas the flexure members were fabricated using a DM-8530-Grey 60 polypropylene like digital material. This material has intermediate properties, in terms of Young's modulus, among the available choice of digital materials. Apart from the Young's modulus, other parameters affecting the magnitude of resulting strain are the structural parameters $e_{1}, e_{2}, r_{1}$ and $w$ of the designed flexible element. To obtain the required range of strain, the structural parameters were varied rather than the material. Having too soft or too stiff material would have reduced the effect of the structural parameters over the variation in the strain produced. Also by varying the structural parameters rather than just the Young's modulus of the material, there were more variables to optimize with. The gages used with the prototype are EA-06-125PC-350LDM dual-pattern gages from Micro-Measurements, which are recommended for use in back-to-back bending applications. They are connected to form a full Wheatstone bridge.

\section{QuASI-STATIC ChaRACTERIZATION OF THE SENSOR}

In this section, an experimental setup for quasi-static analysis of the force sensor is described. The results obtained after the sensor quasi-static characterization are given. This brought to light the hysteresis effect in the force-deformation curve leading to the conclusion that a more elaborate model was necessary for the validation and use of the CSM force sensor.

\section{A. Experimental Setup}

The first experimental setup utilized in quasi-static loading conditions is shown in Fig. 4. It consists of a testing machine from Zwick, GmbH (Z005 THN - Allround Line). The essential components of the experimental setup, namely the cross-head, the calibrated force sensor (XForce HP 50N with an accuracy class 0.5 to ISO 5893 and NF ISO 7500-1) and the prototype CSM force sensor, can be seen in Fig. 4. The cross-head of the testing machine applies unidirectional force on the platform of the CSM along $-\mathbf{z}$. Though extremely accurate, this setup cannot provide arbitrary input force profile such as harmonic excitations. A second experimental setup for

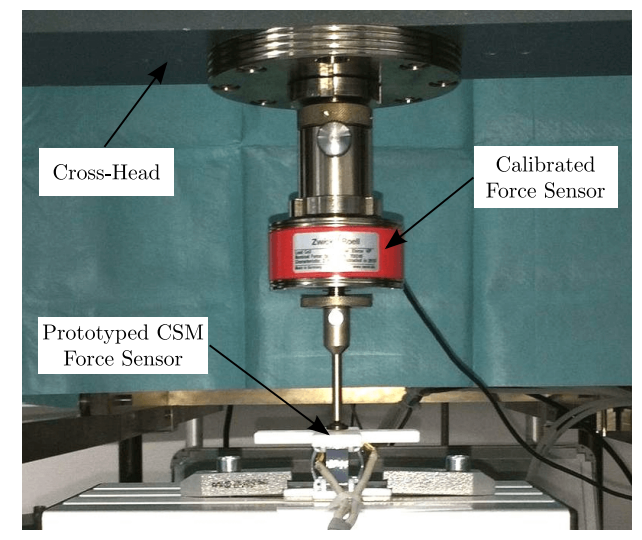

Fig. 4: Experimental setup for quasi-static loadings.

assessing dynamic response of the CSM force sensor will be described in section $\mathrm{V}$. In both experimental setups, the voltage signal from the full Wheatstone bridge of the prototype is sent to a $\mathrm{CPJ} / \mathrm{CPJ} 2 \mathrm{~S}$ analog signal conditioner from Scaime, which amplifies the signal. This amplified signal is in turn acquired by a computer.

\section{B. Quasi-Static Experiments}

A first experiment is carried out to calculate the gain of the sensor under static conditions. Different loads within the range $0.05,1,1.5,2,3,4 \cdots 10 \mathrm{~N}$ are applied to the prototype and each load gives one point of the static characteristics. This static sensor calibration is shown in Fig. 5. A least square fit is applied to obtain the gain and offset required to derive the force value from the electrical measurement. The coefficient of determination for this fit is 0.993 . The obtained static gain is $0.36 \mathrm{~V} / \mathrm{N}$ and the linearity error of the force sensor is calculated to be $4.6 \%$.

In a second experiment, a constant force rate input of $1 \mathrm{~N} / \mathrm{s}$ for the forward and reverse loading is applied. It increases linearly up to $25 \mathrm{~N}$ and then decreases linearly to $0 \mathrm{~N}$. The loading conditions are measured and controlled by the testing machine and its force sensor, whereas the electrical signal of the prototype force sensor is acquired simultaneously. The force applied by the testing machine $v s$ the CSM force sensor output which corresponds to the prototype deformation is plotted in Fig. 6 and shows hysteresis. It can be observed that the CSM force sensor deformation is retarded with respect to the applied force during the reverse loading and that the initial deformation is not totally recovered at the end of the test. These effects are typical of the viscoelastic behavior found in polymer materials [27]. This emphasizes the fact that the approximation of the sensor model to a pure spring does not account for the viscoelastic effects. Therefore a more comprehensive model needs to be used for modeling of the prototype before proceeding to dynamic characterization and final validation of the CSM force sensor.

\section{Viscoelastic Modeling}

An adapted viscoelastic model would lead to a better understanding of the sensor behavior, as compared to a simple 


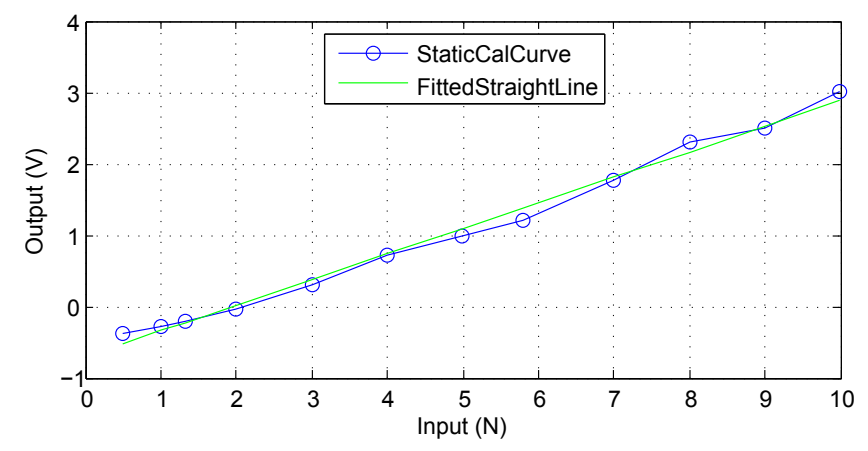

Fig. 5: Static calibration. Reprinted from [22].

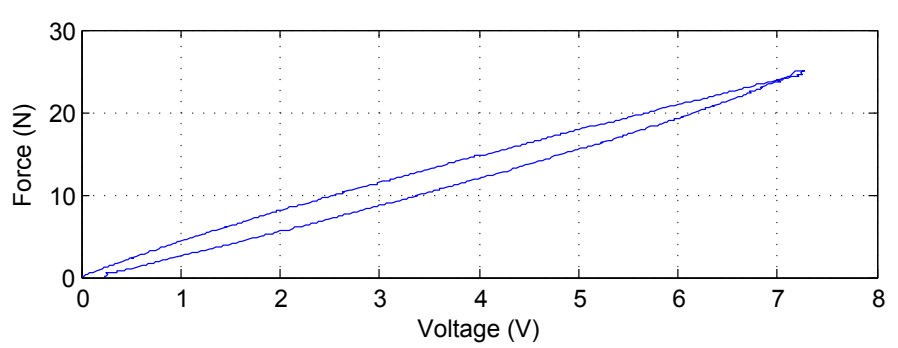

Fig. 6: Force/Voltage plot showing hysteresis. Reprinted from [22].

elastic model. This will also give a compensation method to account for the hysteresis and the time-dependent effects such as drift in the sensor signal value during cyclic loadings, as discussed in the section IV-C.

\section{A. Viscoelastic Model Selection}

A linear viscoelastic model can be described by an ordinary linear differential equation $\chi$ expressed as:

$$
\chi(F, \dot{F}, \cdots, \gamma, \dot{\gamma}, \cdots)=0
$$

where $F$ and $\gamma$ denote the applied force and resulting deformation respectively. There are two main tests one can perform for the identification of the viscoelastic model $\chi$ :

1) Relaxation experiment: In this test the deformation is kept constant while the force levels are measured as a function of time: $\chi\left(F, \dot{F}, \cdots, \gamma=\gamma_{r}\right)=0$.

2) Creep experiment: In this test the force levels are kept constant while the deformation levels are measured as a function of time: $\chi\left(F=F_{c}, \gamma, \dot{\gamma}, \cdots\right)=0$.

Since the output of the CSM force sensor is a voltage corresponding to the deformation $\gamma$, the creep experiment must be used to identify the model $\chi$. This test also allows to investigate the response of the CSM force sensor to a step force input.

Different linear viscoelastic models and their respective time response to a creep test [27] are shown in Fig. 7. The symbols $k_{i}$ and $\eta_{i}$ represent the stiffness and damping coefficients of the models. Even though the basic 2-parameter Maxwell and Kelvin models can describe the time-dependent behavior, their combination with additional dashpots or springs provides overall better results for modeling viscoelastic behavior. For

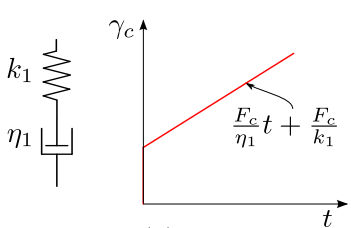

(a) Maxwell

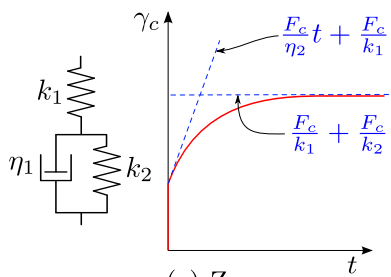

(c) Zener

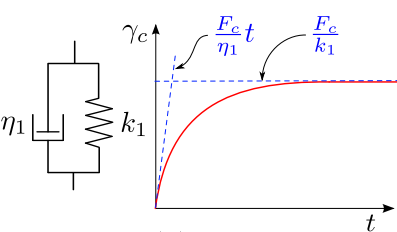

(b) Kelvin

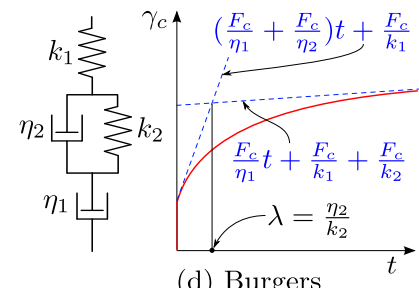

(d) Burgers

Fig. 7: Linear viscoelastic models and their creep response.

(a)

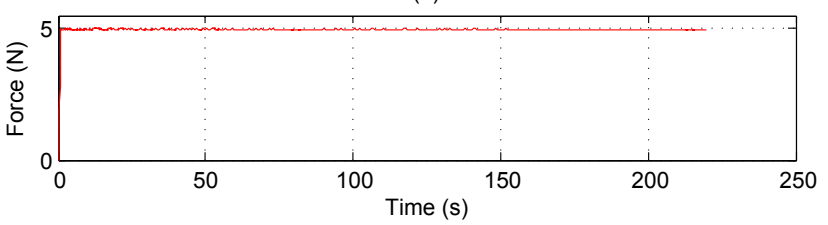

(b)

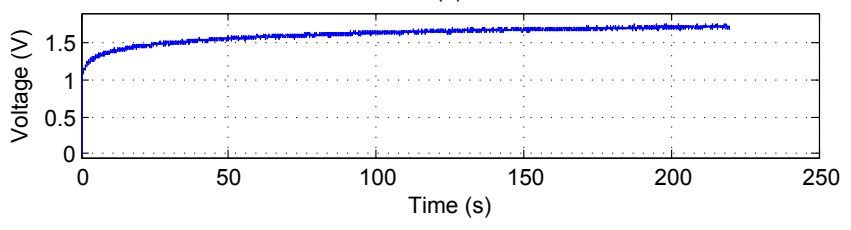

Fig. 8: Creep experiment on the CSM force sensor (a) Input load (b) Output response.

instance, the Zener model also called the standard viscoelastic model enriches the Kelvin model with instantaneous elasticity provided by the $k_{1}$ spring. However, the deformation in the creep response of the Zener model becomes constant as time increases. To account for the fact that the deformation keeps increasing slowly as time increases for most viscoelastic materials, the Burgers model has been introduced. By matching the creep response of the actual prototype with one of these models or some other model, one could obtain an approximate viscoelastic model for the compensation of the output signal of the CSM force sensor. It should be noted here that the deformation $\gamma$ corresponds to the sensor output, which is measured in voltage.

Fig. 8 shows the output of the CSM force sensor as response to the creep test with a constant force input of $F_{c}=5 N$. A careful observation shows a slow increase in the deformation value as the time increases. Hence, Fig. 8(b) matches best with the creep response of the Burgers model with two springs and two dashpots as shown in Fig.7(d). Thus the identified model for describing the behavior of the CSM force sensor is that of the Burgers. 


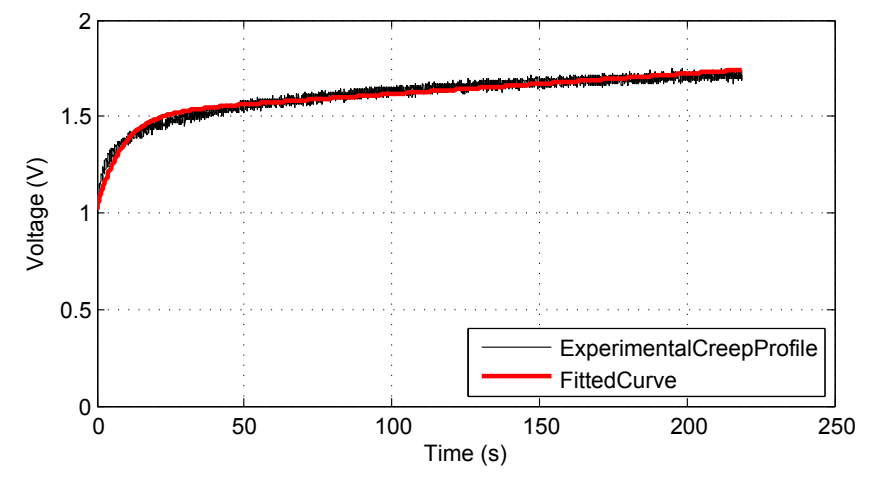

Fig. 9: Fitting of the experimental creep profile with the Burgers model.

\section{B. Identification of the Burgers Model Parameters}

The equation representing the Burgers model $\chi_{\text {burgers }}$ is given by:

$$
F \frac{k_{2}}{\eta_{1}}+\dot{F}\left(1+\frac{k_{2}}{k_{1}}+\frac{\eta_{2}}{\eta_{1}}\right)+\ddot{F} \frac{\eta_{2}}{k_{1}}=k_{2} \dot{\gamma}+\eta_{2} \ddot{\gamma}
$$

Before formulating the compensation law, the four constant parameters $k_{1}, k_{2}, \eta_{1}$ and $\eta_{2}$ in Eqn. (2) need to be identified using the creep response. For a constant input $F=F_{c}$, Eqn. (2) reduces to

$$
F_{c} \frac{k_{2}}{\eta_{1}}=k_{2} \dot{\gamma}_{c}+\eta_{2} \ddot{\gamma}_{c}
$$

This equation can be solved analytically for $\gamma_{c}$ with the initial conditions $\gamma_{c}(0)=\frac{F_{c}}{k_{1}}$ and $\dot{\gamma}_{c}(0)=F_{c}\left(\frac{1}{\eta_{1}}+\frac{1}{\eta_{2}}\right)$ to obtain

$$
\gamma_{c}(t)=F_{c}\left[\frac{1}{k_{1}}+\frac{1}{k_{2}}\left(1-e^{\frac{-k_{2} t}{\eta_{2}}}\right)+\frac{t}{\eta_{1}}\right]
$$

where the three terms in the bracket correspond to i) the instantaneous elasticity due to the $k_{1}$ spring, ii) the retarded or damped elasticity due to the $k_{2}$ spring and $\eta_{2}$ dashpot of the Burgers model, iii) the irrecoverable viscous effect of the $\eta_{1}$ dashpot.

The four coefficients in Eqn. (4) can be obtained through fitting this equation with the output creep profile in Fig. 8(b). The function fittype of Matlab has been used for the fitted curve plot in red in Fig. 9. The coefficient of determination for this curve fitting is 0.902 . As a result, the fitting model parameters $k_{1}, k_{2}, \eta_{1}, \eta_{2}$ are calculated to be:

$$
\begin{array}{ll}
k_{1}=4.8 \mathrm{~N} / \mathrm{V} & k_{2}=10.0 \mathrm{~N} / \mathrm{V} \\
\eta_{1}=4627.94 \mathrm{Ns} / \mathrm{V} & \eta_{2}=85.12 \mathrm{Ns} / \mathrm{V}
\end{array}
$$

As indicated in Fig.7(d), the retardation time $\lambda$ is equal to $\frac{\eta_{2}}{k_{2}}$ and yields $\lambda=8.51 \mathrm{~s}$. Inverse of the static stiffness, the coefficient $k_{s}=\frac{k_{1} k_{2}}{k_{1}+k_{2}}$ represents the static gain which is calculated to be $0.31 \mathrm{~V} / \mathrm{N}$. Hereafter, all the comparison between the uncompensated signal and the compensated sensor signal will be done using this value of the static gain. It is close to the $0.36 \mathrm{~V} / \mathrm{N}$ obtained experimentally from the quasi-static experiment described in section III-B.

\section{Compensation Law for the CSM Force Sensor}

To take into account the viscoelastic behavior, a compensation law needs to be derived from the Burgers model. From Eqn. (2), it is not possible to derive an analytical solution for $F$ given an arbitrary input $\gamma$. Thus a numerical solution needs to be developed to compute $F$ in real time. However, the derivative terms in Eqn. (2) will make the compensation law less stable to high frequency noise. Therefore a different formulation needs to be utilized.

The applied force corresponding to an arbitrary deformation history can be obtained using the Boltzmann superposition principle which reflects the assumed linearity of the viscoelastic behavior [28]. The corresponding equation writes:

$$
F(t)=\gamma(0) G(t)+\int_{0}^{t} G(t-\tau) \dot{\gamma}(\tau) d \tau
$$

where $G(t)=\frac{F_{r}(t)}{\gamma_{r}}$ is the relaxation modulus of the respective viscoelastic model. The equation for deriving the relaxation response $F_{r}(t)$ is obtained from Eqn (2) with a constant input $\gamma=\gamma_{r}$

$$
F_{r} \frac{k_{2}}{\eta_{1}}+\dot{F}_{r}\left(1+\frac{k_{2}}{k_{1}}+\frac{\eta_{2}}{\eta_{1}}\right)+\ddot{F}_{r} \frac{\eta_{2}}{k_{1}}=0 .
$$

The relaxation modulus is derived to be of the form:

$$
G(t)=a_{1} e^{-r_{1} t}+a_{2} e^{-r_{2} t}
$$

where i) $r_{1}$ and $r_{2}$ are the roots of the polynomial $\frac{\eta_{2}}{k_{1}} r^{2}-$ $\left(1+\frac{k_{2}}{k_{1}}+\frac{\eta_{2}}{\eta_{1}}\right) r+\frac{k_{2}}{\eta_{1}}=0$, ii) $a_{1}$ and $a_{2}$ are constant coefficients defined using the initial conditions, namely $G(0)=k_{1}$ and $\dot{G}(0)=-k_{1}^{2}\left(\frac{1}{\eta_{1}}+\frac{1}{\eta_{2}}\right)$. The obtained numerical values are:

$$
\begin{array}{ll}
a_{1}=3.23 \mathrm{~N} / \mathrm{V} & a_{2}=1.57 \mathrm{~N} / \mathrm{V} \\
r_{1}=0.699 \cdot 10^{-3} \mathrm{~s}^{-1} & r_{2}=0.173 \mathrm{~s}^{-1}
\end{array}
$$

The Eqn. (6) in its original form requires the derivative of the input signal which might make it sensitive to noise. Hence, the alternative relation is obtained by using integration by parts:

$$
F(t)=\gamma(t) G(0)-\int_{0}^{t} \dot{G}(t-\tau) \gamma(\tau) d \tau
$$

After substituting Eqn. (8) into Eqn. (10) and after discretizing, the compensation law is obtained to process the sensor signal:

$$
\begin{aligned}
F_{i}= & \gamma_{i} G(0)-a_{1} r_{1} e^{-r_{1} t_{i}} \sum_{j=1}^{i} e^{r_{1} t_{j}} \gamma_{j} T_{s} \\
& -a_{2} r_{2} e^{-r_{2} t_{i}} \sum_{j=1}^{i} e^{r_{2} t_{j}} \gamma_{j} T_{s}
\end{aligned}
$$

where the index $i$ refers to the $i^{\text {th }}$ element in the force or deformation data series. The difference between the $i^{t h}$ and $(i+1)^{t h}$ terms in the time series corresponds to the sampling period $T_{s}$. This form is free from the derivative term, so it would be stable against the noise and higher frequency terms. 


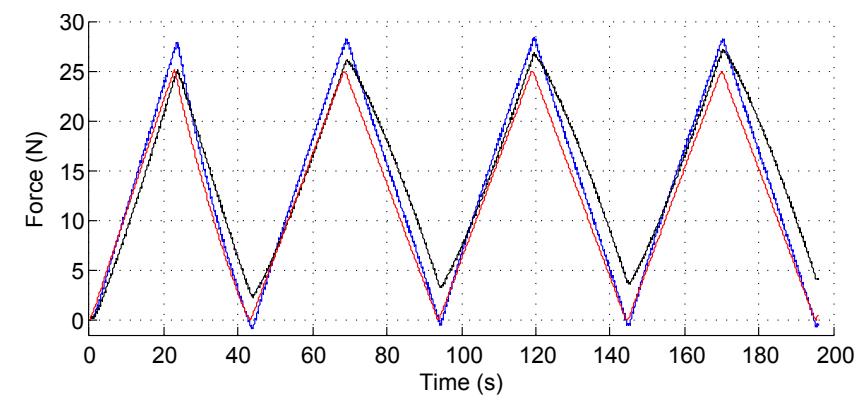

Fig. 10: CSM submitted to a triangle sawtooth force (red): output with (blue) and without compensation (black).

An implementation of the compensation law in Eqn. (11) can be provided as follows:

$$
\begin{array}{lc}
A R_{1}=a_{1} r_{1} T_{s} & A R_{2}=a_{2} r_{2} T_{s} \\
L_{1}=A R_{1} e^{r_{1} t_{1}} \gamma_{1} & M_{1}=A R_{2} e^{r_{2} t_{1}} \gamma_{1} \\
L_{i}=L_{i-1}+A R_{1} e^{r_{1} t_{i}} \gamma_{i} & M_{i}=M_{i-1}+A R_{2} \\
F_{i}=\gamma_{i} k_{1}-e^{-r_{1} t_{i}} L_{i}-e^{-r_{2} t_{i}} M_{i}
\end{array}
$$

This recurrence relation can be used for both offline and real-time processing of the sensor signal. To validate the above compensation law, it was applied to the output signal resulting from cyclic constant force rate experiments. The input triangle sawtooth force profile is given in red in Fig. 10. The output of the CSM force sensor (in black), obtained without compensation, shows a drift of the minimum and maximum values, which is increasing with each cycle. At the end of the fourth cycle, the minimum has increased from 0 to $4 \mathrm{~N}$. The compensated output force profile is shown in blue, where the level of the minimum for each of the four cycles has been brought near to zero values. Also the drift in the maxima of the sensor response has been corrected in the compensated force profile.

In Fig. 11, a comparison of the hysteresis levels is done between the signals with and without compensation. The referred signal is the first cycle of the output profile, shown in Fig. 10, consisting of a forward and reverse loading. In this signal with and without compensation, both force and deformation levels have been normalized by division with the maximum value, so as to allow the relative comparison of the hysteresis levels. The level of hysteresis is calculated to be approximately $20 \%$ and $4.5 \%$ for the uncompensated and the compensated signal, respectively. As a result, there is significant improvement in the hysteresis levels when using this viscoelastic model.

The procedure for the modeling, the identification of the sensor model and the implementation of the compensation law for correction of the force sensor signal is summarized in Fig. 12.

\section{Sensor Resolution}

A change of $0.1 \mathrm{~N}$ was stated in the specification to be detected by the sensor in the event of capsule puncture of the liver during a direct access to the organ. A resolution higher

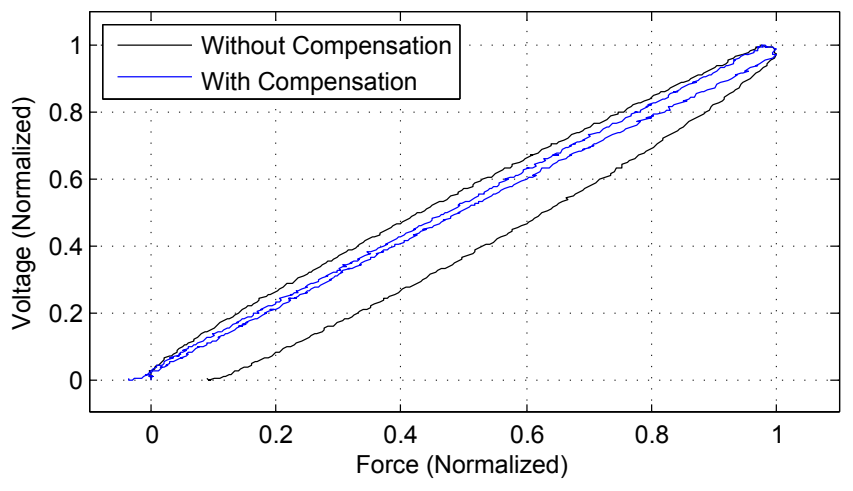

Fig. 11: Hysteresis comparison.

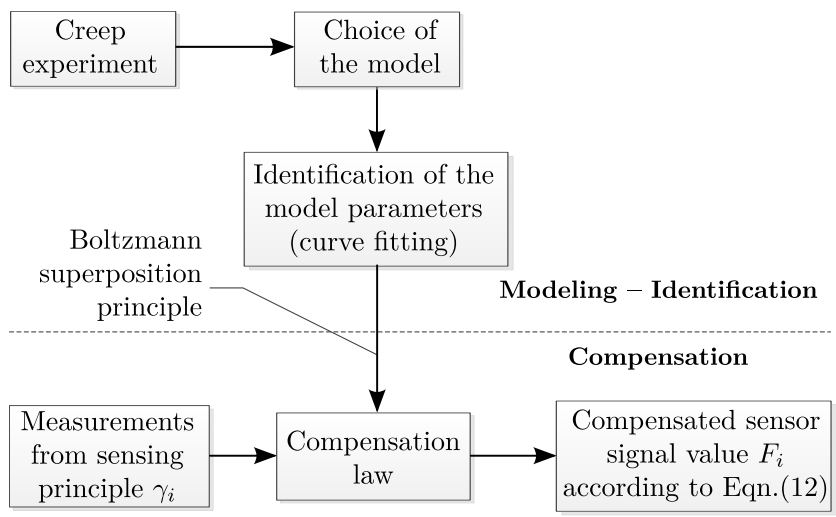

Fig. 12: Procedure for the modeling, the identification of the sensor model and the implementation of the compensation law.

than $0.1 \mathrm{~N}$ would be required, if a very precise measurement of this change needed to be made. However only haptic cues of this event need to be registered which is possible if the sensor is able to detect very small changes in the order of $0.1 \mathrm{~N}$. Thus a resolution of $0.1 \mathrm{~N}$ would be sufficient. A rectangular wave signal of $0.1 \mathrm{~N}$ was applied as an input to determine the prototype resolution. To avoid the issue of loss of contact between the testing machine and the CSM force sensor, a constant preload of $3.75 \mathrm{~N}$ was applied in addition to the cyclic loading. Fig. 13 shows the plots of the input, the output with and without compensation in red, blue and black colors, respectively. In this plot, the constant voltage corresponding to the preload has been subtracted. It can be seen that there is a slow drift in the mean value of the cyclic loading. Since the interest lies in the measured change in sensor value, the output sensor value with compensation is brought down to zero at the beginning of each cycle, as shown in Fig. 14. In response to the input of $0.1 \mathrm{~N}$, the data for $40 \mathrm{~s}$ in the middle of each cycle separated by two dotted black lines as shown in Fig. 14 was used for calculating the mean and standard deviation. The input and output data was recorded with a sampling period of $0.1 \mathrm{~s}$. The average and standard deviation of the changes in sensor values were calculated be $0.08 \mathrm{~N}$ and $0.013 \mathrm{~N}$, respectively. 


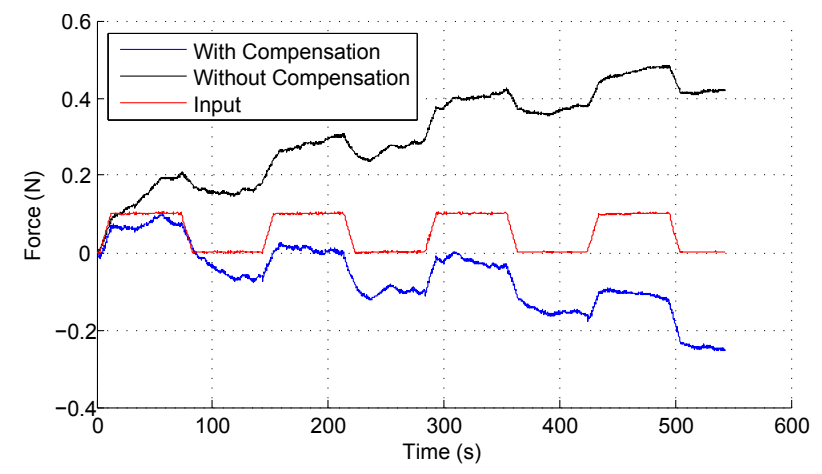

Fig. 13: Test for a resolution of $0.1 \mathrm{~N}$.

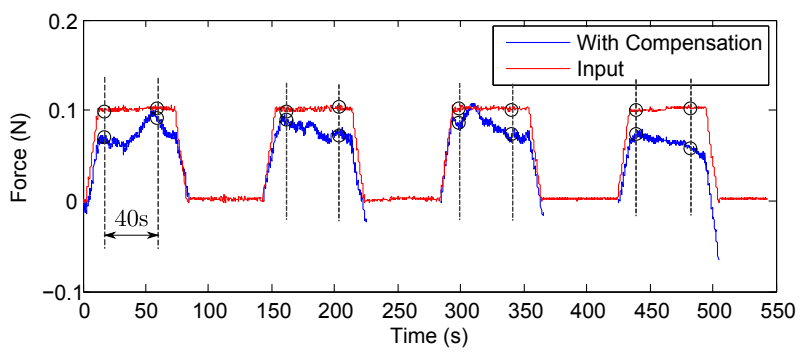

Fig. 14: Relative changes in the sensor value.

\section{Dynamic Characterization of The Force SEnsor}

In this section, a second experimental setup is used to complement the dynamic analysis of the force sensor. The results are given using the compensation model and compared against the results without compensation.

\section{A. Experimental Setup}

To determine the sensor response under arbitrary loadings, the setup shown in Fig. 15 has been utilized. It consists of a two DOF system (X-Y table from Nanomotion) with a calibrated force sensor (Scaime-K1107-20N). Only one DOF of the $\mathrm{X}-\mathrm{Y}$ table is used, so as to apply axial forces to the prototype sensor as an input.

\section{B. Step Response}

The CSM force is submitted to a $5 \mathrm{~N}$ step input and the output is studied. Fig. 16 shows how the compensation improves the dynamic response of the sensor. The rise time of the step response, which corresponds to the time required for rise of the signal to from 10 to $90 \%$ of the step input, was found to be $0.3 \mathrm{~s}$ for the compensated signal. Also, the compensated signal stabilizes after some time whereas the uncompensated signal does not stabilize even after a very long time and shows a small drift with increase in time.

\section{Harmonic Response}

An harmonic analysis was performed over the frequency range $[0.01 ; 10] \mathrm{Hz}$. The vibrations in the setup increased notably at higher frequencies, hence the study was limited to frequency of $10 \mathrm{~Hz}$. Fig. 17 shows the Bode magnitude plot

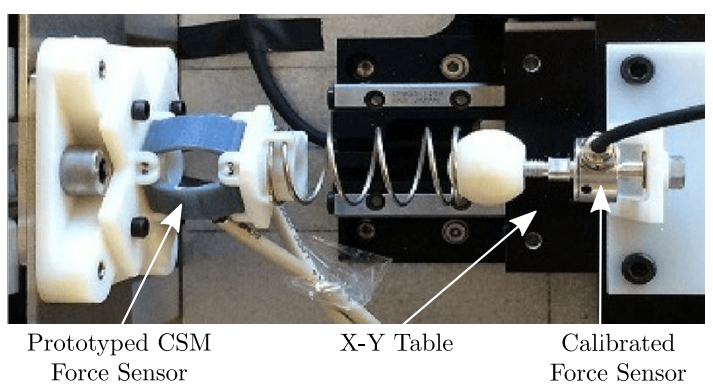

Fig. 15: Experimental setup for dynamic loadings.

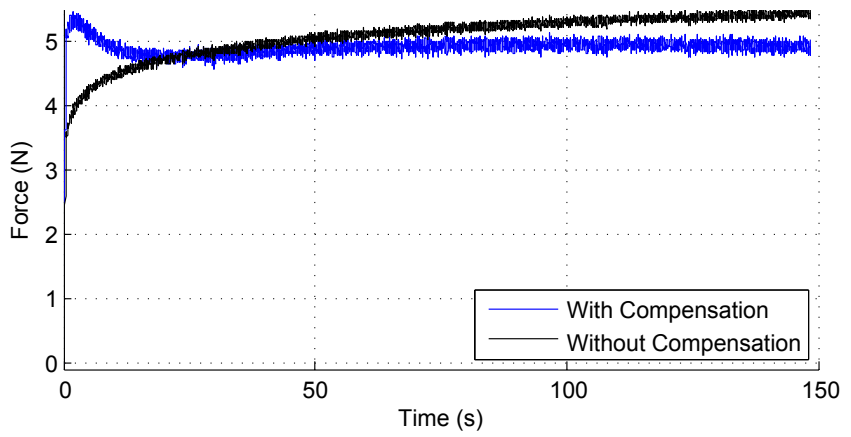

Fig. 16: Response to a step input of $5 \mathrm{~N}$.

with and without compensation. There is a definite improvement in the frequency response at lower frequencies. At higher frequencies, the response seems to be comparable for both as effects due to dashpots are negligible. The offset between the two curves at higher frequencies is due to lower value of the static gain. The bandwidth for the force sensor (measured at $-3 \mathrm{~dB}$ point) without compensation is roughly around $2 \mathrm{~Hz}$, whereas the bandwidth of the sensor without compensation is more than $8 \mathrm{~Hz}$. Though $-3 \mathrm{~dB}$ point is not reached even for frequencies higher than $8 \mathrm{~Hz}$, it is difficult to associate it to the bandwidth of the sensor due to the resonance type behavior around $10 \mathrm{~Hz}$. However, there is an increase in the bandwidth which is due to the upward shift in the response at lower frequencies.

\section{Validation}

A final validation of the prototype force sensor was performed using an input force profile obtained from an actual in vivo needle insertion procedure carried out on an anesthetized swine (Fig. 18). This force profile was earlier presented and used in [7]. The fit between the applied force and the measured force signal is much better with viscoelastic compensation than without compensation. This is reflected in the coefficient of the determination for this fit, which is calculated to be 0.966 and 0.835 with and without compensation, respectively. In Fig. 18, most of the haptic information including the transients which are very important to feel key events such as ruptures of tissues are present, with very limited attenuation. Hence, the developed CSM force sensor can be effectively used for haptic force feedback in a bilateral teleoperation scenario. 


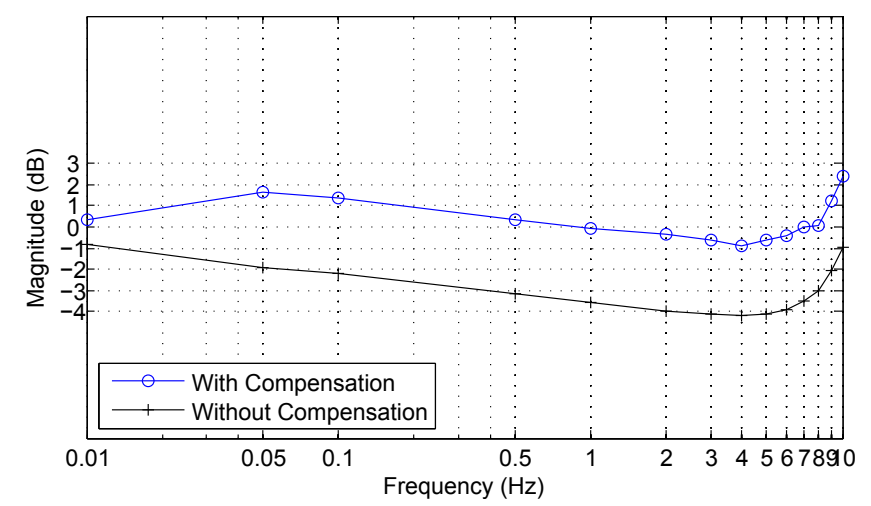

Fig. 17: Bode magnitude plot.

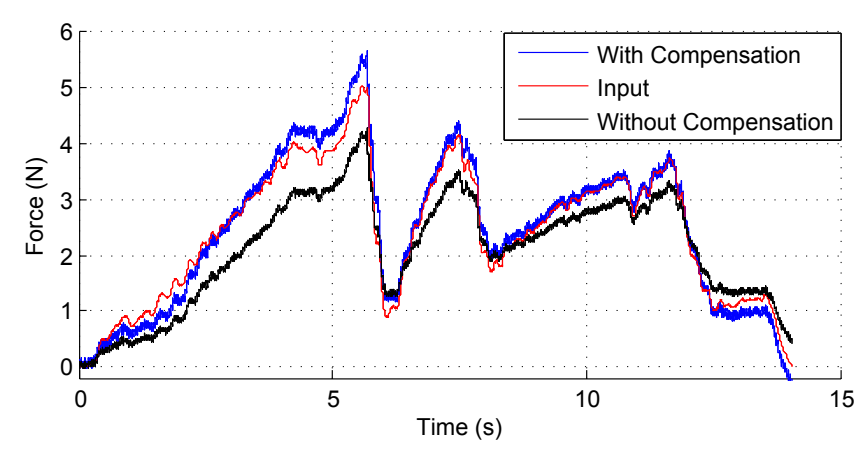

Fig. 18: Response to the experimental force profile as input.

\section{CONCLUSION}

The requirements for the design of a force sensor compatible with CT scanner are clearly laid out in this article. A complete characterization of the force sensor was done through quasistatic and dynamic analysis. A systematic viscoelastic model identification was done to model the time-dependent effects due to the polymer based material of the force sensor. A novel stable, computationally effective compensation law was derived from a linear viscoelastic model to improve the CSM force sensor response. The compensated CSM force sensor signal has much lower levels of the hysteresis and a much improved dynamic response. Through experimental evaluation, the requirements arising from the needle insertion procedures in interventional radiology set out in the beginning of the paper have been shown to be met.

Though the CSM force sensor was developed for CT compatibility and for specific medical procedures, it could be adapted and utilized for wide variety of applications where uni-axial force sensing is a requirement. The viscoelastic model identification through representative creep response presented in the paper can be used to model and compensate for the time-dependent response in sensors utilizing wide variety of polymer based materials. This seems to be a promising alternative to using non-linear models for modeling the viscoelastic effects including hysteresis of the sensors fabricated out of polymer materials. For signals with varying frequency content, compensated signal corrects automatically for the gain of the sensor as compared to using single static or harmonic gain for all type of signals. Future work consists of integration of the developed force sensor in a novel robotic assistant under development and to measure needle insertion forces in medical procedures under in vivo conditions.

\section{ACKNOWLEDGMENT}

The authors acknowledge the support of the Image-guided Hybrid Surgery Institute (IHU Strasbourg) and the Foundation ARC. This work has been sponsored by the French government research program Investissements d'Avenir through the Robotex Equipment of Excellence and Labex CAMI (ANR10-EQPX-44 and ANR-11-LABX-0004).

\section{REFERENCES}

[1] R. Taylor, "A perspective on medical robotics," Proceedings of the IEEE, vol. 94, no. 9, pp. 1652-1664, Sep. 2006.

[2] R. H. Taylor, A. Menciassi, G. Fichtinger, and P. Dario, "Medical robotics and computer-integrated surgery," in Springer Handbook of Robotics, B. S. Prof and O. K. Prof, Eds. Springer Berlin Heidelberg, Jan. 2008, pp. 1199-1222.

[3] D. Stoianovici, C. Kim, G. Srimathveeravalli, P. Sebrecht, D. Petrisor, J. Coleman, S. Solomon, and H. Hricak, "MRI-safe robot for endorectal prostate biopsy," IEEE/ASME Transactions on Mechatronics, vol. 19, no. 4, pp. 1289-1299, Aug. 2014.

[4] J. Gwilliam, M. Mahvash, B. Vagvolgyi, A. Vacharat, D. D. Yuh, and A. Okamura, "Effects of haptic and graphical force feedback on teleoperated palpation," in IEEE International Conference on Robotics and Automation, 2009. ICRA '09, May 2009, pp. 677-682.

[5] A. Talasaz, A. Trejos, and R. Patel, "Effect of force feedback on performance of robotics-assisted suturing," in 2012 4th IEEE RAS EMBS International Conference on Biomedical Robotics and Biomechatronics (BioRob), Jun. 2012, pp. 823-828.

[6] O. Gerovich, P. Marayong, and A. M. Okamura, "The effect of visual and haptic feedback on computer-assisted needle insertion," Computer aided surgery: official journal of the International Society for Computer Aided Surgery, vol. 9, no. 6, pp. 243-249, 2004.

[7] O. Piccin, L. Barbé, B. Bayle, M. d. Mathelin, and A. Gangi, "A force feedback teleoperated needle insertion device for percutaneous procedures," The International Journal of Robotics Research vol. 28, no. 9, pp. 1154-1168, Sep. 2009. [Online]. Available: http://ijr.sagepub.com/content/28/9/1154

[8] A. Okamura, "Methods for haptic feedback in teleoperated robotassisted surgery," The Industrial robot, vol. 31, no. 6, pp. 499-508, Dec. 2004, PMID: 16429611 PMCID: PMC1317565. [Online]. Available: http://www.ncbi.nlm.nih.gov/pmc/articles/PMC1317565/

[9] D. Stoianovici, K. Cleary, A. Patriciu, D. Mazilu, A. Stanimir, N. Craciunoiu, V. Watson, and L. Kavoussi, "AcuBot: a robot for radiological interventions," IEEE Transactions on Robotics and Automation, vol. 19, no. 5, pp. 927-930, Oct. 2003.

[10] C. J. Walsh, J.-A. Shepard, R. Gupta, N. C. Hanumara, and A. H. Slocum, "A patient-mounted, telerobotic tool for CTGuided percutaneous interventions," Journal of Medical Devices, vol. 2, no. 1, pp. 011007-011007, Apr. 2008. [Online]. Available: http://dx.doi.org/10.1115/1.2902854

[11] N. Hungr, C. Fouard, A. Robert, I. Bricault, and P. Cinquin, "Interventional Radiology Robot for CT and MRI Guided Percutaneous Interventions," in Medical Image Computing and Computer-Assisted Intervention - MICCAI 2011, ser. Lecture Notes in Computer Science, G. Fichtinger, A. Martel, and T. Peters, Eds. Springer Berlin Heidelberg, Jan. 2011, no. 6891, pp. 137-144.

[12] S. Shah, A. Kapoor, J. Ding, P. Guion, D. Petrisor, J. Karanian, W. F. Pritchard, D. Stoianovici, B. J. Wood, and K. Cleary, "Robotically assisted needle driver: evaluation of safety release, force profiles, and needle spin in a swine abdominal model," International Journal of Computer Assisted Radiology and Surgery, vol. 3, no. 1-2, pp. 173-179, Jun. 2008. [Online]. Available: http://link.springer.com/article/10.1007/s11548-008-0164-2

[13] R. Gassert, R. Moser, E. Burdet, and H. Bleuler, "MRI/fMRI-compatible robotic system with force feedback for interaction with human motion," IEEE/ASME Transactions on Mechatronics, vol. 11, no. 2, pp. 216-224, Apr. 2006 
[14] R. Gassert, D. Chapuis, H. Bleuler, and E. Burdet, "Sensors for applications in magnetic resonance environments," IEEE/ASME Transactions on Mechatronics, vol. 13, no. 3, pp. 335-344, 2008.

[15] P. Polygerinos, L. Seneviratne, R. Razavi, T. Schaeffter, and K. Althoefer, "Triaxial catheter-tip force sensor for MRI-Guided cardiac procedures," IEEE/ASME Transactions on Mechatronics, vol. 18, no. 1, pp. 386-396, Feb. 2013.

[16] H. Su and G. Fischer, "A 3-axis optical force/torque sensor for prostate needle placement in magnetic resonance imaging environments," in IEEE International Conference on Technologies for Practical Robot Applications, 2009. TePRA 2009, 2009, pp. 5-9.

[17] M. Yip, S. Yuen, and R. Howe, "A robust uniaxial force sensor for minimally invasive surgery," IEEE Transactions on Biomedical Engineering, vol. 57, no. 5, pp. 1008-1011, May 2010.

[18] U.-X. Tan, B. Yang, R. Gullapalli, and J. Desai, "Triaxial MRICompatible fiber-optic force sensor," IEEE Transactions on Robotics, vol. 27, no. 1, pp. 65-74, Feb. 2011.

[19] M. Al Janaideh and P. Krejci, "Inverse rate-dependent Prandtl-Ishlinskii model for feedforward compensation of hysteresis in a piezomicropositioning actuator," IEEE/ASME Transactions on Mechatronics, vol. 18 , no. 5, pp. 1498-1507, Oct. 2013.

[20] G. Palli, G. Borghesan, and C. Melchiorri, "Modeling, identification, and control of tendon-based actuation systems," IEEE Transactions on Robotics, vol. 28, no. 2, pp. 277-290, Apr. 2012.

[21] C. H. Nguyen, G. Alici, and G. Wallace, "An advanced mathematical model and its experimental verification for trilayer conjugated polymer actuators," IEEE/ASME Transactions on Mechatronics, vol. 19, no. 4, pp. 1279-1288, Aug. 2014.

[22] N. Kumar, O. Piccin, L. Meylheuc, L. Barbé, and B. Bayle, "Design, development and preliminary assessment of a force sensor for robotized medical applications," in IEEE/ASME International Conference on Advanced Intelligent Mechatronics, 2014. AIM '14, Jul. 2014 (in press).

[23] B. Maurin, B. Bayle, O. Piccin, J. Gangloff, M. de Mathelin, C. Doignon, P. Zanne, and A. Gangi, "A patient-mounted robotic platform for CTScan guided procedures," IEEE Transactions on Biomedical Engineering, vol. 55, no. 10, pp. 2417-2425, Oct. 2008.

[24] B. Maurin, L. Barbé, B. Bayle, P. Zanne, J. Gangloff, M. Mathelin, A. Gangi, L. Soler, and A. Forgione, "In vivo study of forces during needle insertions," in Perspective in Image-guided Surgery: Proceedings of the Scientific Workshop on Medical Robotics, Navigation, and Visualization (MRNV 2004), 2004, p. 415-422.

[25] A. Graña, A. Sánchez, N. Zemiti, and P. Poignet, "Modelling and control of an ERF-Based needle insertion training platform," in Information Processing in Computer-Assisted Interventions, ser. Lecture Notes in Computer Science, D. Stoyanov, D. L. Collins, I. Sakuma, P. Abolmaesumi, and P. Jannin, Eds. Springer International Publishing, Jan. 2014, no. 8498 , pp. $31-40$

[26] L. L. Howell, Compliant mechanisms. New York: Wiley, 2001.

[27] S. I. Krishnamachari, Applied stress analysis of plastics: a mechanical engineering approach. New York: Van Nostrand Reinhold, 1993. [Online]. Available: http://dx.doi.org/10.1007/978-1-4615-3110-4

[28] H. F. Brinson and L. C. Brinson, Polymer Engineering Science and Viscoelasticity. Springer US, Jan. 2008, ch. Hereditary Integral Representations of Stress and Strain, pp. 201-219. 